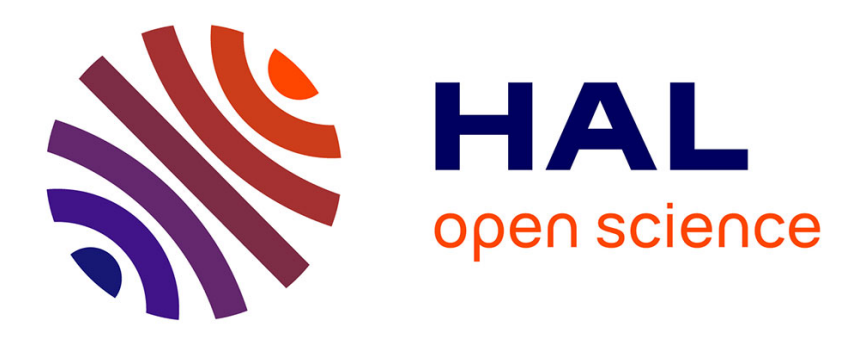

\title{
Localisation conditionnelle de diviseurs
}

Régis de La Bretèche, Gérald Tenenbaum

\section{To cite this version:}

Régis de La Bretèche, Gérald Tenenbaum. Localisation conditionnelle de diviseurs. J. Sander, J. Steuding, R. Steuding. From arithmetic to zeta-functions. Number theory in memory of Wolfgang Schwarz, Springer, 2016. hal-01281749

\section{HAL Id: hal-01281749 https://hal.science/hal-01281749}

Submitted on 2 Mar 2016

HAL is a multi-disciplinary open access archive for the deposit and dissemination of scientific research documents, whether they are published or not. The documents may come from teaching and research institutions in France or abroad, or from public or private research centers.
L'archive ouverte pluridisciplinaire HAL, est destinée au dépôt et à la diffusion de documents scientifiques de niveau recherche, publiés ou non, émanant des établissements d'enseignement et de recherche français ou étrangers, des laboratoires publics ou privés. 
$(22 / 12 / 2015,16 \mathrm{~h} 02)$

\title{
Localisation conditionnelle de diviseurs
}

\author{
Régis de la Bretèche \& Gérald Tenenbaum \\ À la mémoire de Wolfgang Schwarz, \\ pour la délicatesse et la distinction
}

\begin{abstract}
In support of a still little known, general principle according to which the structure of the set of prime factors of an integer is statistically governed by its actual cardinal, we show that, given any $\varepsilon>0$, the conditional probability that an integer with exactly $k$ prime factors has a divisor in a dyadic interval ] $y, 2 y$ ] approaches 0 as $y \rightarrow \infty$ if $2^{(1+\varepsilon) k}<\log y$ while it remains larger than a strictly positive constant when $2^{(1-\varepsilon) k}>\log y$.
\end{abstract}

Key-words. Distribiution of divisors, integers with $k$ prime factors.

Résumé. À l'appui d'un principe général peu connu selon lequel la structure de la suite des facteurs premiers d'un entier est statistiquement gouvernée par son cardinal global, nous montrons que, pour tout $\varepsilon>0$, la probabilité conditionnelle pour qu'un entier ayant $k$ facteurs premiers possède un diviseur dans un intervalle dyadique $] y, 2 y]$ tend vers 0 lorsque $y \rightarrow \infty$ si $2^{(1+\varepsilon) k}<\log y$ mais qu'elle demeure plus grande qu'une constante strictement positive si $2^{(1-\varepsilon) k}>\log y$.

Mots-clefs. Répartition des diviseurs, entiers ayant $k$ facteurs premiers.

Classification AMS 2010. Principale 11N25.

\section{Introduction}

La localisation de diviseurs dans des intervalles dyadiques pose un délicat problème de crible : aucune relation de divisibilité ne lie les entiers d'un intervalle ]$y, 2 y](y \geqslant 1)$, mais les ensembles de multiples de deux éléments quelconques ne sont généralement pas indépendants.

Considérons la densité $\varepsilon_{y}$ de l'ensemble $\mathcal{H}_{y}$ des entiers naturels ayant au moins un diviseur $d$ tel que $y<d \leqslant 2 y$. Besicovitch [3] a établi en 1934 que

$$
\liminf _{y \rightarrow \infty} \varepsilon_{y}=0
$$

et en a déduit une remarquable réfutation de la conjecture en vigueur à l'époque selon laquelle tout ensemble de multiples admettrait une densité naturelle.

Erdős [5] a montré en 1936 que $\varepsilon_{y} \rightarrow 0$. Cependant, la détermination du véritable ordre de grandeur de $\varepsilon_{y}$ a longtemps constitué un véritable défi pour les spécialistes. Précisant une estimation du second auteur [10], Ford [7] a montré en 2008 que l'on a

$$
\varepsilon_{y} \asymp \frac{1}{(\log y)^{\delta}\left(\log _{2} y\right)^{3 / 2}} \quad(y \geqslant 3)
$$

où l'on a posé $\delta:=1-\left(1+\log _{2} 2\right) / \log 2 \approx 0.08607$ et où, ici et dans la suite, nous désignons par $\log _{k}$ la $k$-ième itérée de la fonction logarithme. Nous renvoyons le lecteur à l'article de synthèse [14] pour une mise en perspective plus complète de ce problème et de ses ramifications. 
La valeur de l'exposant $\delta$ peut être devinée facilement : la croissance normale des facteurs premiers d'un entier $n$ étant doublement exponentielle (voir par exemple [6] et le chapitre 1 de [8]), on peut approcher, en première analyse, le nombre des diviseurs de $n$ n'excédent pas $2 y$ par $2^{\omega(n, y)}$, où $\omega(n, y)$ désigne le nombre des facteurs premiers n'excédant pas $y$; si l'on fait l'hypothèse que tous les intervalles dyadiques reçoivent un quota statistique de diviseurs, on est amené à conjecturer que les entiers de $\mathcal{H}_{y}$ sont caractérisés par la condition $2^{\omega(n, y)} \gg \log y$. Or la densité de l'ensemble des entiers $n$ tels que $\omega(n, y) \geqslant\left(\log _{2} y\right) / \log 2$ vaut classiquement $1 /(\log y)^{\delta+o(1)}$ — voir par exemple [12], ch. II.6.

Nous nous intéressons ici à un phénomène particulier, connu des spécialistes mais qui, à notre connaissance, n'a jamais été clairement mis en évidence dans la littérature : la structure locale de la suite des facteurs premiers d'un entier est statistiquement gouvernée par son cardinal global. À l'appui de ce principe, et dans l'intention d'en fournir une illustration emblématique, nous nous proposons d'examiner la probabilité conditionnelle pour qu'un entier $n$ ayant $k$ facteurs premiers possède un diviseur dans un intervalle ]y,2y] donné. Conformément à l'heuristique développée plus haut, la conclusion qualitative de nos résultats est que cette probabilité est minorée par une constante positive lorsque $\omega(n):=\omega(n, n)$ dépasse sensiblement $\left(\log _{2} n\right) / \log 2$ alors qu'elle tend vers 0 dans le cas contraire.

Désignons par $\mathcal{E}_{k}(x)$ l'ensemble des entiers $n \leqslant x$ tels que $\omega(n)=k$, par $\pi_{k}(x)$ son cardinal, et par $H_{k}(x, y)$ le nombre des entiers $n$ de $\mathcal{E}_{k}(x)$ qui ont au moins un diviseur dans $] y, 2 y]$. Nos résultats sont contenus dans les deux énoncés suivants.

Théorème 1.1. Soient $R>0, \beta>1 / \log 2$. Il existe des constantes positives $c, K$, dépendant au plus de $R$ et $\delta$, telles que l'on ait

$$
c \pi_{k}(x) \leqslant H_{k}(x, y) \leqslant(1-c) \pi_{k}(x)
$$

uniformément pour $2 \leqslant y \leqslant x^{1 / K}, \beta \log _{2} x \leqslant k \leqslant R \log _{2} x$. De plus, l'inégalité de droite est valable pour $1 \leqslant k \leqslant R \log _{2} x$.

Cet énoncé induit deux questions naturelles, sur lesquelles nous espérons revenir dans un prochain travail :

(a) est-il possible de remplacer, dans les hypothèses, la constante $\beta$ par une quantité tendant vers $1 / \log 2$ ?

(b) l'encadrement (1.1) est-il valable pour de plus grandes valeurs de $k$, par exemple $R \log _{2} x<k \leqslant(\log x)^{1-\varepsilon}$ ?

Comme annoncé, la valeur $1 / \log 2$ est critique pour la minoration de (1·1). Dans toute la suite, nous utilisons les notations

$$
u:=\frac{\log x}{\log y}, \quad \mathfrak{g}(x, y):=\sqrt{\frac{\log u}{\left(\log _{2} y\right) \log _{2} x}} \quad(x \geqslant y \geqslant 3)
$$


Théorème 1.2. Soit $\xi(x) \rightarrow \infty$. Sous les conditions

$$
\xi(x) \leqslant y \leqslant \sqrt{x}, \quad k \leqslant \frac{\log _{2} x}{\log 2}\{1-\xi(x) \mathfrak{g}(x, y)\}
$$

nous avons uniformément

$$
H_{k}(x, y)=o\left(\pi_{k}(x)\right)
$$

Remarques. (i) Nous avons

$$
\frac{1 / \sqrt{2}+o(1)}{\log _{2} x} \leqslant \mathfrak{g}(x, y) \leqslant \frac{1}{\sqrt{\log _{2} y}}
$$

dans le domaine $(1 \cdot 3)$.

(ii) Des majorations effectives de $H_{k}(x, y)$ peuvent être aisément déduites de la démonstration donnée au paragraphe 5 . Nous nous sommes cantonnés ici à dégager le phénomène qualitatif.

\section{Répartition des diviseurs des entiers de $\mathcal{E}_{k}(x)$}

Le résultat suivant fait l'objet du lemme 1 de [13].

Lemme 2.1. Soient $A>0, B>0$ et $f$ une fonction arithmétique multiplicative positive ou nulle telle que

$$
\sum_{p^{\nu} \leqslant x} f\left(p^{\nu}\right) \log p^{\nu} \leqslant A x \quad(x \geqslant 2), \quad \sum_{p} \sum_{\nu \geqslant 2} \frac{f\left(p^{\nu}\right)}{p^{\nu}} \leqslant B .
$$

Alors on a, uniformément pour $k \geqslant 1, x \geqslant 2$,

$$
\sum_{n \in \mathcal{E}_{k}(x)} f(n) \ll \frac{A x}{\log x} \frac{\left(\sum_{p \leqslant x} f(p) / p+B\right)^{k-1}}{(k-1) !},
$$

où la constante implicite est absolue. En particulier, pour toute constante $R>0$, nous avons, uniformément sous la condition $1 \leqslant k \leqslant R \log _{2} x$,

$$
\sum_{n \in \mathcal{E}_{k}(x)} f(n) \ll \pi_{k}(x) \exp \left\{\varrho \sum_{p \leqslant x} \frac{f(p)-1}{p}\right\}
$$

avec $\varrho:=(k-1) / \log _{2} x$ et où la constante implicite dépend au plus de $A, B, R$. 
Dans toute la suite, nous nous donnons des paramètres $x \geqslant 2, y \in[2, \sqrt{x}]$, et $L \in\left[1, \log _{2} y\right]$. Soit $\mathfrak{s}_{L}:=\exp \exp L(L>0)$. Pour chaque entier $n$, nous écrivons

$$
n_{L}:=\prod_{\substack{p \leqslant \mathfrak{s}_{L} \\ p^{\nu} \| n}} p^{\nu} .
$$

Enfin, il est commode d'introduire la fonction $Q: \mathbb{R}^{+} \rightarrow \mathbb{R}^{+}$définie par

$$
Q(\alpha):=\alpha \log \alpha-\alpha+1 .
$$

Notons que $Q(\alpha) \geqslant 0$ avec égalité si, et seulement si, $\alpha=1$.

Lemme 2.2. Soient $R>0, x \geqslant 3$ et $1 \leqslant k \leqslant R \log _{2} x$. Posant

$$
L_{1}:=\min \left(L, \log _{2} x-L\right)
$$

et définissant implicitement $\kappa \in] 0, R]$ par $k=\kappa \log _{2} x$, nous avons, uniformément pour $1 \leqslant L \leqslant \log _{2} x, 1 \leqslant \xi \leqslant \sqrt{L_{1}}$,

$$
\left|\left\{n \in \mathcal{E}_{k}(x):\left|\omega\left(n_{L}\right)-\kappa L\right|>\kappa \xi \sqrt{L_{1}}\right\}\right| \ll_{R} \pi_{k}(x) \mathrm{e}^{-\kappa \xi^{2} / 3},
$$

où l'on peut remplacer $\xi^{2} / 3$ par $\xi^{2} / 2$ si $\xi \leqslant L_{1}^{1 / 6}$.

Démonstration. Supposons dans un premier temps que $L \leqslant \frac{1}{2} \log _{2} x$, et donc $L_{1}=L$. Posons $s_{1}=1-\xi / \sqrt{L_{1}}, s_{2}=1+\xi / \sqrt{L_{1}}$. Le cardinal à majorer n'excède pas

$$
\sum_{1 \leqslant j \leqslant 2} \sum_{n \leqslant x} s_{j}^{\omega\left(n_{L}\right)-s_{j} \kappa L} \ll \pi_{k}(x)\left\{\mathrm{e}^{-\kappa Q\left(s_{1}\right) L}+\mathrm{e}^{-\kappa Q\left(s_{2}\right) L}\right\} \ll \pi_{k}(x) \mathrm{e}^{-\kappa \xi^{2} / 3},
$$

où la première estimation découle du Lemme 2.1 et la seconde de l'évaluation

$$
\frac{1}{3} s^{2} \leqslant Q(1+s) \leqslant \frac{1}{2} s^{2} \leqslant Q(1-s) \quad(0 \leqslant s \leqslant 1) .
$$

L'assertion relative au cas $\xi \leqslant L^{1 / 6}$ résulte du développement

$$
Q(1+s)=\frac{1}{2} s^{2}+O\left(s^{3}\right)
$$

au voisinage de $s=0$.

Lorsque $L>\frac{1}{2} \log _{2} x$, et donc $L_{1}=\log _{2} x-L$, nous observons que la condition sur $\omega\left(n_{L}\right)$ apparaissant dans (2.4) équivaut à $\left|\omega(n)-\omega\left(n_{L}\right)-(k-\kappa L)\right|>\kappa \xi \sqrt{L_{1}}$. On peut alors procéder similairement au cas précédent, en considérant les premiers de $\left.] \mathfrak{s}_{L}, x\right]$. 
Lemme 2.3. Soit $R>0, x \geqslant 3$ et $1 \leqslant k \leqslant R \log _{2} x$. On définit implicitement $\kappa \in] 0, R]$ par $k=\kappa \log _{2} x$. Nous avons, uniformément pour $1 \leqslant L \leqslant \log _{2} x, T>0$,

$$
\left|\left\{n \in \mathcal{E}_{k}(x): \log n_{L}>T \mathrm{e}^{L}\right\}\right| \ll \pi_{k}(x) \mathrm{e}^{-T / 2} .
$$

Démonstration. Nous pouvons supposer $L$ assez grand puisque le résultat est trivial lorsque $L$ est borné. Posons $\alpha:=\frac{1}{2} \mathrm{e}^{-L}$. Alors la fonction $n \mapsto n_{L}^{\alpha}$ est multiplicative et, puisque $\sqrt{\mathrm{e}}<2$, satisfait les hypothèses du Lemme 2.1 pour des constantes absolues convenables $A$ et $B$. La quantité à estimer n'excède pas

$$
\sum_{n \in \mathcal{E}_{k}(x)}\left\{n_{L} / \exp \left(T \mathrm{e}^{L}\right)\right\}^{\alpha}=\mathrm{e}^{-T / 2} \sum_{n \in \mathcal{E}_{k}(x)} n_{L}^{\alpha} .
$$

Le Lemme 2.1 fournit aisément que la dernière somme est $\ll \pi_{k}(x)$. L'estimation souhaitée en résulte.

Nous posons encore

$$
\begin{aligned}
\omega_{\vartheta}(m) & :=\sum_{p \mid m, p \leqslant \exp (1 /|\vartheta|)} 1 \quad(m \geqslant 1, \vartheta \neq 0), \\
\omega(n ; s, t) & :=\sum_{p \mid n, s<\log _{2} p \leqslant t} 1 \quad(0<s \leqslant t), \\
\sigma(t) & :=t-\sqrt{t \log t} \quad(t>1) .
\end{aligned}
$$

Le résultat suivant est énoncé, à fins d'applications ultérieures, sous une forme significativement plus précise que nécessaire pour le présent travail.

Lemme 2.4. Soient $\left.R>0, \varepsilon \in] 0, \frac{1}{2}\right], x \geqslant 3,1 \leqslant k \leqslant R \log _{2} x, 1 \leqslant L \leqslant \log _{2} x$. On définit implicitement $\kappa \in] 0, R]$ par $k=\kappa \log _{2} x$. Nous avons

$$
\min _{1 /\left(\varepsilon \mathrm{e}^{L}\right) \leqslant|\vartheta| \leqslant 2} \frac{\omega\left(n_{L}\right)-\omega_{\vartheta}\left(n_{L}\right)}{\sigma(L+\log |\vartheta|)}>\kappa
$$

pour tous les entiers $n$ de $\mathcal{E}_{k}(x)$ sauf au plus $\ll \pi_{k}(x) /\{\log (1 / \varepsilon)\}^{1 / 4}$. La constante implicite dépend au plus de $R$.

Démonstration. Considérons un entier $n$ contrevenant à $(2 \cdot 5)$. Il existe alors $\left.\vartheta \in] 1 /\left(\varepsilon \mathrm{e}^{L}\right), 2\right]$ tel que, posant $j_{\vartheta}:=\lfloor L+\log |\vartheta|\rfloor$, et donc $j_{\vartheta}>\log (1 / \varepsilon)-1$, l'on puisse écrire, pour tout $v \in] 0,1]$,

$$
1 \leqslant v^{\omega_{\vartheta}\left(n_{L}\right)-\omega\left(n_{L}\right)-\kappa \sigma\left(j_{\vartheta}\right)}<v^{-\kappa-\kappa\left(j_{\vartheta}\right)} v^{\omega\left(n ; L-j_{\vartheta}, L\right)} .
$$

Ainsi, le nombre $R_{x}$ des entiers exceptionnels satisfait

$$
R_{x} \leqslant \sum_{\log (1 / \varepsilon)-1<j \leqslant L} v_{j}^{-\kappa\{1+\sigma(j)\}} \sum_{n \in \mathcal{E}_{k}(x)} v_{j}^{\omega(n ; L-j, L)},
$$


pour tout choix de $\left.\left.v_{j} \in\right] 0,1\right]$. Nous pouvons employer le Lemme 2.1 pour majorer la somme intérieure. Elle est

$$
\ll \pi_{k}(x) \mathrm{e}^{-\kappa\left(1-v_{j}\right) j} .
$$

Pour le choix $v_{j}:=1-\sqrt{(\log j) / j}$, nous obtenons le facteur sommé dépendant de $j$ est $\ll \pi_{k}(x) j^{-5 / 4}$. Il suit

$$
R_{x} \ll \sum_{j>\log (1 / \varepsilon)-1} \frac{\pi_{k}(x)}{j^{5 / 4}} \ll \frac{\pi_{k}(x)}{\{\log (1 / \varepsilon)\}^{1 / 4}} .
$$

Soit $\mathscr{L}(m):=\cup_{d \mid m}\left(\log d+\left[-\frac{1}{2}, 0[)\right.\right.$. Nous désignons par $\lambda(m)$ la mesure de Lebesgue de $\mathscr{L}(m)$ et posons

$$
\tau(m, \vartheta):=\sum_{d \mid m} d^{i \vartheta} \quad\left(m \in \mathbb{N}^{*}, \vartheta \in \mathbb{R}\right) .
$$

Le résultat suivant a été établi dans [11].

Lemme 2.5. Pour chaque entier naturel $m$, nous avons

$$
\lambda(m) \int_{-2}^{2} \frac{|\tau(m, \vartheta)|^{2}}{\tau(m)^{2}} \mathrm{~d} \vartheta \geqslant \frac{1}{3 \pi} .
$$

Lemme 2.6. Soit $R>0$. Nous avons, uniformément pour $x \geqslant 3,1 \leqslant k \leqslant R \log _{2} x$ et $\vartheta \in \mathbb{R}^{*}$,

$$
\sum_{n \in \mathcal{E}_{k}(x)} \frac{\left|\tau\left(n_{L}, \vartheta\right)\right|^{2}}{2^{\omega\left(n_{L}\right)+\omega_{\vartheta}\left(n_{L}\right)}} \ll \pi_{k}(x)\{\log (3+|\vartheta|)\}^{2 \kappa} .
$$

Démonstration. Appliquons à nouveau le Lemme 2.1. Le membre de gauche de (2.7) est

$$
\ll \pi_{k}(x) \exp \left\{\kappa \sum_{\log (1 /|\vartheta|)<\log _{2} p \leqslant L} \frac{\cos (\vartheta \log p)}{p}\right\} .
$$

La somme en $p$ peut être estimée par sommation d'Abel à l'aide du théorème des nombres premiers, comme au lemme III.4.13 de [12]. En estimant trivialement la contribution éventuelle des nombres premiers $p$ tels que

$$
\exp (1 /|\vartheta|)<p \leqslant \exp \left\{\log ^{2}(3+|\vartheta|)\right\},
$$

nous obtenons qu'elle n'excède pas $2 \log _{2}(3+|\vartheta|)+O(1)$. Cela fournit bien $(2 \cdot 7)$

Lemme 2.7. Soient $R>0, \beta>1 / \log 2, \delta:=\beta \log 2-1$. Pour tous $x \geqslant 2, \varepsilon>0$, $k \in \mathbb{N}$, tels que

$$
\beta \log _{2} x \leqslant k \leqslant R \log _{2} x,
$$

le nombre $M_{k}(x ; \varepsilon)$ des entiers $n$ de $\mathcal{E}_{k}(x)$ tels que $\lambda\left(n_{L}\right) \leqslant \varepsilon \mathrm{e}^{L}$ satisfait

$$
M_{k}(x ; \varepsilon) \ll \pi_{k}(x)\left\{\varepsilon^{\delta / 2}+\varepsilon^{\kappa Q(1-\delta / 3)}\right\} .
$$

La constante implicite dépend au plus de $R$ et $\beta$. 
Démonstration. D'après $(2 \cdot 6)$, un entier $n$ compté dans $M_{k}(x ; \varepsilon)$ satisfait nécessairement

$$
3 \pi \varepsilon \mathrm{e}^{L} \int_{-2}^{2} \frac{\left|\tau\left(n_{L}, \vartheta\right)\right|^{2}}{\tau\left(n_{L}\right)^{2}} \mathrm{~d} \vartheta \geqslant 1 .
$$

Comme la contribution à l'intégrale des nombres réels $\vartheta$ tels que $|\vartheta| \leqslant \mathrm{e}^{-L} /(12 \pi \varepsilon)$ est trivialement $\leqslant \frac{1}{2}$, il suit

$$
\varepsilon_{1} \mathrm{e}^{L} \int_{\mathrm{e}^{-L} / \varepsilon_{1}}^{2} \frac{\left|\tau\left(n_{L}, \vartheta\right)\right|^{2}}{\tau\left(n_{L}\right)^{2}} \mathrm{~d} \vartheta \geqslant 1,
$$

où, pour simplifier l'écriture, nous avons posé $\varepsilon_{1}:=12 \pi \varepsilon$.

Maintenant, nous observons que, quitte à écarter un ensemble d'entiers $n$ de cardinal majoré par le membre de gauche de $(2 \cdot 8)$, le Lemme 2.4 nous permet de supposer que

$$
\min _{1 /\left(\varepsilon_{1} \mathrm{e}^{L}\right) \leqslant|\vartheta| \leqslant 2} \frac{\omega\left(n_{L}\right)-\omega_{\vartheta}\left(n_{L}\right)}{L+\log |\vartheta|}>\frac{1+\delta / 2}{\log 2},
$$

et donc

$$
2^{\omega\left(n_{L}\right)-\omega_{\vartheta}\left(n_{L}\right)}>\left(\vartheta \mathrm{e}^{L}\right)^{1+\delta / 2} .
$$

Cela implique

$$
\int_{\mathrm{e}^{-L} / \varepsilon_{1}}^{2} \frac{\left|\tau\left(n_{L}, \vartheta\right)\right|^{2}}{\tau\left(n_{L}\right)^{2}} \mathrm{~d} \vartheta \leqslant \int_{\mathrm{e}^{-L} / \varepsilon_{1}}^{2} \frac{\left|\tau\left(n_{L}, \vartheta\right)\right|^{2}}{2^{\omega\left(n_{L}\right)+\omega_{\vartheta}\left(n_{L}\right)}} \frac{\mathrm{d} \vartheta}{\left(\vartheta \mathrm{e}^{L}\right)^{1+\delta / 2}} .
$$

En tenant compte de $(2 \cdot 9)$, nous obtenons

$$
\begin{aligned}
M_{k}(x ; \varepsilon) & \leqslant \varepsilon_{1} \int_{\mathrm{e}^{-L} / \varepsilon_{1}}^{2} \sum_{\substack{n \leqslant x \\
\omega(n)=k}} \frac{\left|\tau\left(n_{L}, \vartheta\right)\right|^{2}}{2^{\omega\left(n_{L}\right)+\omega_{\vartheta}\left(n_{L}\right)}} \frac{\mathrm{d}\left(\vartheta \mathrm{e}^{L}\right)}{\left(\vartheta \mathrm{e}^{L}\right)^{1+\delta / 2}}+O\left(\pi_{k}(x) \varepsilon^{\kappa Q(\alpha)}\right) \\
& \ll \pi_{k}(x)\left\{\varepsilon^{\delta / 2}+\varepsilon^{\kappa Q(\alpha)}\right\},
\end{aligned}
$$

d'après le Lemme 2.6.

\section{Entiers de $\mathcal{E}_{\boldsymbol{k}}(x)$ sans petit facteur premier}

Désignons par $P^{-}(n)$ (resp. $\left.P^{+}(n)\right)$ le plus petit (resp. le plus grand) facteur premier d'un entier générique $n>1$ avec la convention $P^{-}(1)=\infty$ (resp. $\left.P^{+}(1)=1\right)$. Nous posons alors

$$
\pi_{k}(x, y):=\left|\left\{n \in \mathcal{E}_{k}(x): P^{-}(n)>y\right\}\right| .
$$

Dans toute cette section, nous notons systématiquement $x=y^{u}$ lorsque $2 \leqslant y \leqslant x$.

Le résultat suivant a été établi dans [9] (lemme 3.2) comme conséquence très affaiblie de formules asymptotiques dues à Balazard [2] (lemme 5) et Alladi [1] (théorème 7). 
Lemme 3.1. Soit $R>0$. Il existe $u_{0}=u_{0}(R)>1$ tel que l'on ait uniformément pour $u \geqslant u_{0}(R), 1 \leqslant k \leqslant R \log u$,

$$
\pi_{k}(x, y) \asymp \frac{x}{\log x} \frac{(\log u)^{k-1}}{(k-1) !} .
$$

Lemme 3.2. Soient $c_{1}, c_{2}$ des constantes telles que $0<c_{1}<c_{2}$. Il existe $\varepsilon_{1}=\varepsilon_{1}\left(c_{1}, c_{2}\right)>0$ tel que l'on ait, uniformément pour $1 \leqslant z^{c_{1}} \leqslant y \leqslant z \leqslant x^{\varepsilon_{1}}$, $1 \leqslant d \leqslant \sqrt{x}$, et $c_{1} \log u \leqslant k \leqslant c_{2} \log u$,

$$
\pi_{k-1}(x / d, z) \asymp \pi_{k}(x, y) / d .
$$

Démonstration. Cela résulte immédiatement de $(3 \cdot 1)$.

\section{Preuve du Théorème $\mathbf{1 . 1}$}

Commençons par établir la minoration. Nous retenons la notation $\kappa:=k / \log _{2} x$ introduite précédemment.

Nous pouvons supposer $y \geqslant y_{0}$ où $y_{0}$ est une constante arbitrairement choisie. En effet, dans le cas contraire, on a $H_{k}(x, y) \geqslant \pi_{k}(x / m, y) \gg \pi_{k}(x, y)$ avec, par exemple, $m=1+[y] \in] y, 2 y]$;

Soit $\varepsilon$ une constante absolue positive assez petite, dont nous préciserons la valeur ultérieurement. Nous posons $L:=\log _{2} y^{\varepsilon}$, conservons la notation $(2 \cdot 3)$, et considérons l'ensemble

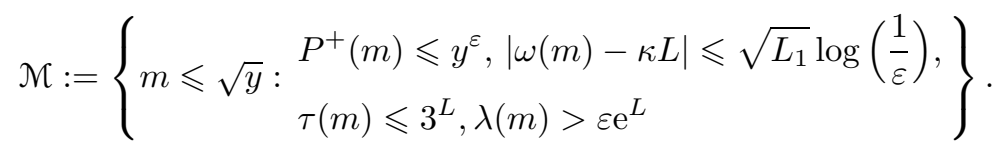

Les Lemmes 2.2, 2.3 et 2.7 garantissent que, pour $\varepsilon$ convenablement choisi, $x>x_{0}(\varepsilon)$, et $K=K(\varepsilon)$ assez grand, on a $n_{L} \in \mathcal{M}$ pour au moins $\frac{1}{2} \pi_{k}(x)$ entiers $n$ de $\mathcal{E}_{k}(x)$. En décomposant chacun de ces entiers sous la forme $m h$ avec $P^{-}(h)>y^{\varepsilon}$, il suit

$$
\frac{1}{2} \pi_{k}(x) \leqslant \sum_{m \in \mathcal{M}} \pi_{k-\omega(m)}\left(x / m, y^{\varepsilon}\right) .
$$

Considérons alors les entiers $n$ de $\mathcal{E}_{k}(x)$ qui peuvent s'écrire sous la forme $m p h$ avec $m \in \mathcal{M}, \log y-\log p \in \mathscr{L}(m), P^{-}(h)>2 y$. La seconde condition implique l'existence d'un diviseur $d$ de $m$ tel que

$$
\log d-\frac{1}{2} \leqslant \log y-\log p<\log d
$$

donc $d p \in] y, 2 y]$. Comme on a nécessairement

$$
\log p>\log y-\log m \geqslant \frac{1}{2} \log y>\log y^{\varepsilon},
$$

la décomposition $n=m p h$, lorsqu'elle existe, est unique. 
Posant $\mathscr{G}(y, m):=\left\{t \in \mathbb{R}^{+}: \log (y / t) \in \mathscr{L}(m)\right\}$, nous pouvons donc écrire

$$
H_{k}(x, y) \geqslant \sum_{m \in \mathcal{M}} \sum_{p \in \mathscr{G}(y, m)} \pi_{k-1-\omega(m)}(x / p m, 2 y) .
$$

Notant à présent $u:=(\log x) / \log 2 y$, nous avons

$$
k-\omega(m)=k-\kappa L+O_{\varepsilon}\left(\sqrt{L_{1}}\right)=\kappa \log u+O_{\varepsilon}\left(\sqrt{L_{1}}\right),
$$

d'après la définition de $\mathcal{M}$. Lorsque $y \leqslant x^{1 / K}$, où $K=K(\varepsilon)$ est une constante convenable, cela implique $k-\omega(m) \asymp \log u$. Nous sommes donc en mesure d'appliquer le Lemme 3.2 avec $d=p$ pour déduire que, pour tous les couples $(m, p)$ de la sommation de $(4 \cdot 2)$, nous avons

$$
\pi_{k-1-\omega(m)}(x / p m, 2 y) \asymp_{\varepsilon} \pi_{k-\omega(m)}\left(x / m, y^{\varepsilon}\right) / p .
$$

À ce stade, nous observons que $\mathscr{G}(y, m)$ est, pour chaque $m \in \mathcal{M}$, une réunion d'au plus $\tau(m) \leqslant(\log y)^{2}$ intervalles de longueur logarithmique totale $\lambda(m)$ et dont les bornes ont des logarithmes de l'ordre de $\log y$. Nous avons donc

$$
\sum_{p \in \mathscr{G}(y, m)} \frac{1}{p} \gg \frac{1}{\log y} \sum_{p \in \mathscr{G}(y, m)} \frac{\log p}{p} \gg \frac{\lambda(m)}{\log y}+O\left(\frac{\log y}{\mathrm{e}^{\sqrt{\log y}}}\right) \gg 1 .
$$

En reportant dans $(4 \cdot 2)$, il suit

$$
H_{k}(x, y) \gg_{\varepsilon} \sum_{m \in \mathcal{M}} \pi_{k-\omega(m)}\left(\frac{x}{m}, y^{\varepsilon}\right) \sum_{p \in \mathscr{G}(y, m)} \frac{1}{p} \gg \sum_{m \in \mathcal{M}} \pi_{k-\omega(m)}\left(\frac{x}{m}, y^{\varepsilon}\right) \gg \pi_{k}(x),
$$

où la dernière minoration résulte de $(4 \cdot 1)$.

Établissons maintenant la majoration de $(1 \cdot 1)$. À cette fin, nous considérons le nombre $J_{k}(x, y)$ des entiers $n$ de $\mathcal{E}_{k}(x)$ tels que $n_{L} \leqslant y$, avec à présent $L:=\log _{2} 2 y$. Nous avons évidemment

$$
\pi_{k}(x)-H_{k}(x, y) \geqslant J_{k}(x, y)
$$

Nous allons montrer que

$$
J_{k}(x, y) \gg \pi_{k}(x)
$$

Chaque entier de la forme $n=m h$ avec $m \leqslant y, P^{-}(h)>2 y$ et $\omega(m)+\omega(h)=k$ est compté dans $J_{k}(x, y)$. On peut donc écrire

$$
J_{k}(x, y) \geqslant \sum_{m \leqslant y} \pi_{k-\omega(m)}(x / m, 2 y) .
$$


Retenons seulement dans la sommation les entiers $m$ tels que

$$
|\omega(m)-\kappa L| \leqslant L_{1}
$$

Nous avons alors $k-\omega(m) \ll \log u=\log _{2} x-\log _{2} y$ sous l'hypothèse $y \leqslant x^{1 / K}$ dès que la constante $K$ est convenablement choisie. Par $(3 \cdot 1)$ il suit

$$
J_{k}(x, y) \gg \frac{x}{\log x} \sum_{\substack{m \leqslant y \\|\omega(m)-\kappa L| \leqslant L_{1}}} \frac{(\log u)^{k-\omega(m)-1}}{(k-\omega(m)-1) ! m} .
$$

Une sommation d'Abel permet de déduire des estimations connues de $\pi_{k}(x)$ (cf., par exemple, [12], chapitre II.6) que l'on a, pour $|j-\kappa L| \leqslant L_{1}$,

$$
\sum_{\substack{m \leqslant y \\ \omega(m)=j}} \frac{1}{m} \asymp_{\varepsilon} \frac{\left(\log _{2} y\right)^{j}}{j !}
$$

En reportant dans $(4 \cdot 4)$, il suit

$$
J_{k}(x, y) \gg \frac{x}{\log x} \frac{\left(\log _{2} x\right)^{k-1}}{(k-1) !} \sum_{|j-\kappa L| \leqslant L_{1}}\left(\begin{array}{c}
k-1 \\
j
\end{array}\right)(1-\vartheta)^{k-1-j} \vartheta^{j}
$$

avec $\vartheta:=\left(\log _{2} y\right) / \log _{2} x$, de sorte que $\kappa L=k \vartheta$ et, pour un choix convenable des constantes, $L_{1} \geqslant 2 \sqrt{\vartheta(1-\vartheta) k}$. Un résultat classique sur les sommes partielles du binôme (voir par exemple le théorème 6.1 de [4]) implique que la somme en $j$ est $\gg 1$. Nous obtenons donc $(4 \cdot 3)$, ce qui achève la démonstration du Théorème 1.1 .

\section{Preuve du Théorème $\mathbf{1 . 2}$}

Nous supposons dans toute la suite $y_{0} \leqslant y \leqslant \sqrt{x}$, où $y_{0}$ désigne une constante assez grande.

Chaque entier $n$ compté dans $H_{k}(x, y)$ admet une représentation sous la forme $n=m d$ avec $d \in] y, 2 y]$. Posons alors

$$
\begin{aligned}
& r=(m, d), \quad m=m_{0} r_{1} r, \quad d=d_{0} r_{2} r, \quad r_{1} r_{2} \mid r^{\infty} \\
& a=\omega\left(m_{0}\right), \quad b=\omega\left(d_{0}\right), \quad j:=a+b \leqslant k,
\end{aligned}
$$

de sorte que $\omega(r)=k-j$.

Il suit

$$
H_{k}(x, y) \leqslant \sum_{\substack{j \leqslant k \\ a+b=j}} \sum_{\substack{r \geqslant 1, r_{1} \geqslant 1, r_{2} \geqslant 1 \\ r_{1} r_{2} \mid r\left(r^{\infty} \\ \omega(r)=k-j\right.}} \sum_{\substack{y / r r_{2}<d \leqslant 2 y / r r_{2} \\ \omega\left(d_{0}\right)=b}} \sum_{\substack{m_{0} \leqslant x / d_{0} r_{1} r_{2} r^{2} \\ \omega\left(m_{0}\right)=a}} 1 .
$$


On montre facilement que la contribution correspondant à la condition supplémentaire $r_{1} r>x^{1 / 4}$ est $\ll \pi_{k}(x) / \log x$ : en effet, une majoration triviale des sommes en $m_{0}$ et $d_{0}$ fournit une borne

$$
\ll \sum_{\substack{r \geqslant 1, r_{1} \geqslant 1, r_{2} \geqslant 1 \\ r_{1} r_{2} \mid r^{\infty} \\ x^{1 / 4}<r_{1} r \leqslant x}} \frac{x}{r_{1} r_{2} r^{2}} \ll \sum_{\substack{r \leqslant x \\ r_{1} r_{2} \mid r^{\infty}}} \frac{x^{7 / 8}}{\sqrt{r_{1}} r_{2} r^{3 / 2}} \ll \sum_{r \geqslant 1} \frac{x^{7 / 8}}{r \varphi(r)} \prod_{p \mid r}\left(1-\frac{1}{\sqrt{p}}\right) \ll x^{7 / 8} .
$$

Lorsque $r_{1} r \leqslant x^{1 / 4}$, des estimations classiques (cf., par exemple, [12], chapitre II.6), impliquent que la double somme intérieure est

$$
\ll \frac{x\left(\log _{2} x\right)^{a-1}\left(\log _{2} y\right)^{b-1}}{r^{2} r_{1} r_{2}(a-1) !(b-1) !(\log 3 y / r) \log x} .
$$

Par sommation sur $a$ et $b$, nous obtenons

$$
\begin{aligned}
H_{k}(x, y) & \ll x \sum_{j \leqslant k} \sum_{\substack{r \geqslant 1, r_{1} \geqslant 1, r_{2} \geqslant 1 \\
r_{1} r_{2} \mid r^{\infty} \\
\omega(r)=k-j}} \frac{\left(\log _{2} x+\log _{2} y\right)^{j-2}}{r^{2} r_{1} r_{2}(j-2) ! \log x(\log 3 y / r)} \\
& \ll x \sum_{j \leqslant k} \sum_{\substack{r \geqslant 1 \\
\omega(r)=k-j}} \frac{\left(\log _{2} x+\log _{2} y\right)^{j-2}}{\varphi(r)^{2}(j-2) ! \log x(\log 3 y / r)} \\
& \ll \frac{\pi_{k}(x)}{\log y}\left(1+\frac{\log _{2} y}{\log _{2} x}\right)^{k}=\frac{2^{k} \pi_{k}(x)}{\log y}\left(1-\frac{\log u}{2 \log _{2} x}\right)^{k},
\end{aligned}
$$

sous l'hypothèse $k \ll \log _{2} x$.

Il vient

$$
H_{k}(x, y) \ll \frac{2^{k} \pi_{k}(x) u^{-k / 2 \log _{2} x}}{\log y}=\frac{2^{k} \pi_{k}(x) u^{1-\kappa / 2}}{\log x}
$$

où nous avons conservé la notation $\kappa=k / \log _{2} x$.

La majoration souhaitée (1.4) est donc acquise dès que

$$
k \leqslant \frac{\log _{2} x}{\log 2}-\frac{1-1 / \log 4}{\log 2} \log u-\xi(x) .
$$

Cela fournit la conclusion annoncée lorsque, disons, $\log y>\mathrm{e}^{-\xi^{2}} \log x$.

Nous pouvons donc supposer dans la suite que $u>\mathrm{e}^{\xi^{2}}$. Choisissons $L:=\log _{2} y$ et conservons la notation $(2 \cdot 3)$. Nous pouvons supposer sans perte de généralité que la fonction $\xi=\xi(x)$ apparaissant dans l'hypothèse $(1 \cdot 3)$ satisfait également $\xi \leqslant \sqrt{L_{1}}$. Soit $\kappa_{1}=\left(1+\xi \sqrt{L_{1}} / L\right) \kappa$. En vertu du Lemme 2.2, quitte à écarter un nombre acceptable d'entiers exceptionnels, nous pouvons supposer que

$$
\omega\left(n_{L}\right) \leqslant \kappa_{1} L
$$


De même, nous déduisons du Lemme 2.3, que l'on peut supposer

$$
n_{L} \leqslant x_{1}:=x^{\exp \left(-\xi^{2} / 2\right)} .
$$

Les entiers restants comptés dans $H_{k}(x, y)$ admettent une représentation sous la forme $n=d t m$, avec les conditions

$$
y<d \leqslant 2 y, \quad P^{+}(t) \leqslant y, \quad t \leqslant x_{1}, \quad P^{-}(m)>y .
$$

Soit $H_{k}^{*}(x, y)$ le nombre de tels entiers $n$ n'excédant pas $x$ et tels que $\omega(n)=k$. Nous avons

$$
H_{k}^{*}(x, y) \leqslant \sum_{j \leqslant \kappa_{1} L} \sum_{y<d \leqslant 2 y} \sum_{\begin{array}{c}
t \leqslant x_{1} \\
P^{+}(t) \leqslant y \\
\omega(d t)=j
\end{array}} \sum_{\begin{array}{c}
m \leqslant x / d t \\
\omega(m)=k-j \\
P^{-}(m)>y
\end{array}} 1 .
$$

La somme intérieure relève du du Lemme 2.1. Elle est

$$
\ll \frac{x}{d t \log x} \frac{\left(\log _{2} x-\log _{2} y+B\right)^{k-j-1}}{(k-j-1) !},
$$

où $B$ est une constante absolue convenable. Posons alors $d=r d_{1}, t=r t_{1}$, avec $\left(d_{1}, t_{1}\right)=1$. Nous avons $\omega\left(d_{1}\right)+\omega\left(t_{1}\right) \leqslant j$, donc

$$
\begin{aligned}
\sum_{y<d \leqslant 2 y} & \sum_{\substack{t \leqslant x_{1} \\
P^{+}(t) \leqslant y \\
\omega(d t)=j}} \frac{1}{d t} \ll \sum_{r \leqslant 2 y} \frac{1}{r^{2}} \sum_{a+b \leqslant j} \sum_{\begin{array}{c}
a / r<d_{1} \leqslant 2 y / r \\
\omega\left(d_{1}\right)=a
\end{array}} \frac{1}{d_{1}} \sum_{\substack{t_{1} \leqslant x_{1} \\
\omega\left(t_{1}\right)=b}} \frac{1}{t_{1}} \\
& \ll \sum_{r \leqslant 2 y} \frac{1}{r^{2}} \sum_{a+b \leqslant j} \frac{\left(\log _{2} y\right)^{a+b-1}}{(a-1) ! b !(\log 3 y / r)} \ll \sum_{r \leqslant 2 y} \frac{1}{r^{2}} \sum_{c \leqslant j-1} \frac{\left(2 \log _{2} y\right)^{c}}{c ! \log (3 y / r)} \\
& \ll \frac{1}{\log y} \sum_{c \leqslant j} \frac{\left(2 \log _{2} y\right)^{c}}{c !} \ll \frac{\left(2 \log _{2} y\right)^{j}}{j ! \log y} .
\end{aligned}
$$

Nous avons utilisé ici les estimations classiques de $\pi_{\ell}(v)$ pour estimer les sommes en $d_{1}$ et $t_{1}$. Pour la dernière majoration, nous avons fait appel au résultat bien connu stipulant que, puisque $j \leqslant \kappa_{1} \log _{2} y$ avec $\kappa_{1} \leqslant 1 / \log 2<2$, la dernière somme en $c$ est dominée par le terme d'indice $c=j$.

Il suit finalement

$$
\begin{aligned}
H_{k}^{*}(x, y) & \ll \frac{x}{(\log x) \log y} \sum_{j \leqslant \kappa_{1} L} \frac{\left(2 \log _{2} y\right)^{j}}{j !} \frac{\left(\log _{2} x-\log _{2} y+B\right)^{k-j-1}}{(k-j-1) !} \\
& \ll \frac{x 2^{\kappa_{1} \log _{2} y}}{(\log x) \log y} \sum_{j \leqslant k} \frac{\left(\log _{2} y\right)^{j}}{j !} \frac{\left(\log _{2} x-\log _{2} y+B\right)^{k-j-1}}{(k-j-1) !} \\
& \ll \pi_{k}(x)(\log y)^{\kappa_{1} \log 2-1} .
\end{aligned}
$$


On vérifie alors que $(\log y)^{\kappa_{1} \log 2-1}$ tend vers 0 dès que

$$
k \leqslant \frac{\log _{2} x}{\log 2}\left(1-\frac{\xi \sqrt{L_{1}}}{L \log 4}\right)
$$

Comme

$$
\sqrt{\frac{L_{1}}{L}} \asymp \sqrt{\frac{\log u}{\left(\log _{2} y\right) \log _{2} x}}=\mathfrak{g}(x, y),
$$

nous obtenons le résultat souhaité, quitte à altérer la fonction arbitraire $\xi$.

\section{Bibliographie}

[1] K. Alladi, The distribution of $\nu(n)$ in the sieve of Eratosthenes, Quart. J. Math. Oxford (2), 33 (1982), 129-148.

[2] M. Balazard, Unimodalité de la distribution du nombre de diviseurs premiers d'un entier, Ann. Inst. Fourier, Grenoble 40, 2 (1990), 255-270.

[3] A.S. Besicovitch, On the density of certain sequences, Math. Annalen 110 (1934), 336-341.

[4] C. Dartyge \& G. Tenenbaum Sommes des chiffres de multiples d'entiers, Ann. Inst. Fourier (Grenoble), 55, 7 (2005), 2423-2474.

[5] P. Erdős, A generalization of a theorem of Besicovitch, J. London Math. Soc. 11 (1936), 92-98.

[6] P. Erdős, On the distribution function of additive functions, Ann. of Math. 47 (1946), 1-20.

[7] K. Ford, The distribution of integers with a divisor in a given interval. Ann. of Math. (2) 168, $\mathrm{n}^{\mathrm{O}} 2$ (2008), 367-433.

[8] R.R. Hall \& G. Tenenbaum, Divisors, Cambridge tracts in mathematics 90, Cambridge University Press (1988, paperback ed. 2008).

[9] A. Raouj, A. Stef \& G. Tenenbaum, Mesures quadratiques de la proximité des diviseurs, Math. Proc. Camb. Phil. Soc. 150 (2011), 73-96.

[10] G. Tenenbaum, Sur la probabilité qu'un entier possède un diviseur dans un intervalle donné, Compositio Math. 51 (1984), 243-263.

[11] G. Tenenbaum, Sur une question d'Erdős et Schinzel, in : A. Baker, B. Bollobás, A. Hajnal (eds.), A Tribute to Paul Erdős, Cambridge University Press (1990), 405-443.

[12] G. Tenenbaum, Introduction à la théorie analytique et probabiliste des nombres, 4-ième éd., coll. Échelles, Belin, 2015, 592 pp.

[13] G. Tenenbaum, A rate estimate in Billingsley's theorem for the size distribution of large prime factors, Quart. J. Math. (Oxford) 51 (2000), 385-403.

[14] G. Tenenbaum, Some of Erdős' unconventional problems in number theory, thirty-four years later, in : L. Lovász I.Z. Ruzsa, V. T. Sós (eds), Erdős Centennial volume, Bolyai Society Mathematical Studies 25 (2013), 651-681.

Régis de la Bretèche

Institut de Mathématiques de Jussieu

UMR 7586 Université Paris Diderot-Paris 7

Université Paris Diderot-Paris 7
Sorbonne Paris Cité, UMR 7586

Institut de Mathématiques de Jussieu-PRG

Case 7012, F-75013 Paris

France
Gérald Tenenbaum

Institut Élie Cartan

Université de Lorraine

BP 70239

54506 Vandœuvre-lès-Nancy Cedex

France 